\title{
Bisphosphonates in pediatric rheumatology: A review
}

\begin{abstract}
Bisphosphonates are osteoclast inhibiting agents that have been used in adult osteoporosis for the last three decades. Success with their earliest reported indication in children for osteogenesis imperfecta has lead to their use for secondary osteoporosis in rheumatological disorders and inflammatory bone diseases in this age group besides some other indications. This review aims to discuss the current usage of these molecules in pediatric rheumatological diseases, the mechanism of action and evidence base for these, adverse effects, monitoring, and controversies surrounding their use.
\end{abstract}

Keywords: bisphosphonates • pediatric rheumatology • osteoporosis

\section{Introduction}

Bisphosphonates, previously known as diphosphonates, are chemically stable analogues of inorganic pyrophosphate (PPi). Studies on effect of bisphosphonates on bones came from the observation that PPi circulates in the body as its natural water softener. The earliest use of bisphosphonates, over 100 years ago, was based on their ability to sequester calcium from water thus leading to their use in prevention of scaling in domestic and industrial water. Only in the last 40 years, they have been used to treat disorders of calcium metabolism in humans, etidronate being the first compound ever used [1]. Bisphosphonates inhibit bone resorption by being selectively taken up and adsorbed to mineral surfaces in bone, and inhibiting the function of osteoclasts, thus their usefulness in disorders that cause bone weakening. They are extensively used for treatment of bone fragility disorders such as Osteogenesis imperfecta (OI) as well as primary and secondary osteoporosis in children. They also have proven efficacy in inflammatory conditions involving the bone, such as Chronic Recurrent Multifocal Osteomyelitis (CRMO) spectrum disorders and pain syndromes, with unclear mechanisms of action. This review aims to focus on the indications of bisphosphonates specific to pediatric rheumatological disorders, rationale for their use, pharmacokinetics and pharmacodynamics relevant to the pediatric population, dosage and preparations, monitoring and adverse effects, and finally, controversies surrounding their use.

\section{Mechanism of action}

Bisphosphonates are pyrophosphate-derived medications that inhibit osteoclastic function, primarilyworking by disruption of the mevalonate pathway involved in osteoclastogenesis. The mechanisms leading to decreased osteoclastic function are:

- inhibition of osteoclast recruitment;

- inhibition of osteoclastic adhesion;

- shortening of the life span of osteoclasts; and

- inhibition of osteoclast activity.

A secondary action on inhibition of calcification by inhibiting formation and aggregation of calcium phosphate crystals, blocking the transformation of amorphous calcium phosphate into hydroxyapatite and delaying the aggregation of apatite crystals, leads to their clinical usefulness in treatment of ectopic calcification such as calcinosis of Juvenile dermatomyositis (JDM) [2]. Preliminary studies of Pamidronate in Ankylosing spondylitis provided evidence for the fact that bisphosphonates reduce inflammation by affecting the regulation of pro and anti-inflammatory cytokines and this may explain the relief of symptoms and inflammation with bisphosphonates in CRMO

\author{
Anita Dhanrajani*1 \& Raju P. \\ Khubchandani ${ }^{2}$ \\ 'Hospital for Sick Chidlren, Toronto, \\ Canada \\ Jaslok Hospital and Research Centre, \\ Mumbai, India
}

*Author for correspondence: anitadhanrajani@gmail.com 
[3]. In CRMO, osteoclastic activity in an acidic microenvironment leads to destruction of bone and thereby damage to the primary afferent nerve fibres, causing pain. Another potential hypothesis explaining pain relief with bisphosphonates in CRMO is that they neutralize acidic environment, thus inactivating osteoclasts [4]. Studies in children show that bisphosphonates do not reduce bone growth, trabecular bone formation or periosteal bone formation (modelling). Reduced bone resorption and ongoing bone growth and modelling results in the significant increase in bone mass and strength observed when bisphosphonates are administered to the growing child [5].

\section{Preparations and Dosage}

Intravenous pamidronate is the most commonly prescribed bisphosphonate in pediatrics and most data on efficacy and safety of pamidronate comes from studies on Osteogenesis imperfecta. Other bisphosphonate preparations with their doses and routes of administration [5,6] are listed in Table 1.

\section{Pharmacokinetics}

Knowledge of pharmacokinetics is essential for appropriate dosage and minimizing side effects. Bisphosphonates are generally poorly absorbed in humans due to their poor lipophilic nature. Nitrogen-containing bisphosphonates (such as PamidronateandAlendronate) haveanabsorption of about $0.7 \%$, whereas bisphosphonates without a nitrogen atom in their side chain (example Etidronate) have a gastrointestinal absorption of $2-2.5 \%$, thus explaining the preferential routes of administration for these compounds. Bisphosphonates are distributed widely in the body, mainly in bone but also in kidney, spleen, and liver. Intravenous bisphosphonates disappear rapidly from plasma with a half life of 1-2 hours. In the bone, they are taken up by osteoclasts or embedded in the bone for continuing bone formation. The main mechanism of elimination of bisphosphonates is by renal excretion [6]. Pamidronate urinary excretion after the cessation of long-term treatment with daily oral administration has been shown to be detectable up to 8 years after the cessation of treatment. There is no relation between the cumulative dose and excretion of the drug in urine, however the renal excretion decreases over time [7]. This may explain the stabilization of BMD in patients long after discontinuation of the drug, but also raises concerns in the pediatric population, specifically girls of child-bearing age, as the long-term effects of bisphosphonates on fetuses have not been evaluated.

\section{Indications and Rationale}

\section{Secondary osteoporosis}

ISCD Pediatric osteoporosis task force position 2013 [8] has defined osteoporosis in children as "clinically significant fracture history along with low BMD ( $Z$ score less than or equal to -2 SD below normal for age and sex)". Osteoporosis in children can be either due to an intrinsic skeletal defect (primary) or secondary to a variety of diseases such as neuromuscular, chronic inflammatory conditions, malignancies, endocrine disorders, or their treatment. Pediatric rheumatological diseases such as Juvenile idiopathic arthritis (JIA), systemic lupus erythematosus (SLE) and others have been shown to be associated with low bone mineral density (BMD) and resultant vertebral and extremity fractures. Low BMD in these conditions is common even prior to starting treatment. It has been shown that up to $30 \%$ of pre-pubertal JIA patients can have low total BMD before initiating treatment with steroids [9]. Uncontrolled ongoing inflammation, with resultant elevated cytokine levels, immobility with reduced mechanical loading on bone, impeded Vitamin $\mathrm{D}$ absorption due to sunscreen use in SLE, high-

Table 1. Bisphosphonate preparations, dose, and route of administration.

\begin{tabular}{|c|c|c|}
\hline Name & Route & Dose and schedule \\
\hline Etidronate & Oral and Intravenous & $5-40 \mathrm{mg} / \mathrm{kg} /$ day \\
\hline Clodronate & Oral and Intravenous & $2 \mathrm{mg} / \mathrm{kg} / \mathrm{day}$ \\
\hline Pamidronate & Intravenous & $0.5-1 \mathrm{mg} / \mathrm{kg} /$ day for 3 days every $2-6$ months \\
\hline Neridronate & Intravenous & $1-2 \mathrm{mg} / \mathrm{kg} /$ day every $3-6$ months \\
\hline Alendronate & Oral & $1-2 \mathrm{mg} / \mathrm{kg} /$ week \\
\hline Ibandronate & Intravenous & $2 \mathrm{mg} \mathrm{every} 3$ months \\
\hline Risedronate & Oral & $2 \mathrm{mg} / \mathrm{kg} /$ week \\
\hline Zoledronate & Intravenous & $0.015-0.05 \mathrm{~g} / \mathrm{kg}$ every 3-6 months \\
\hline
\end{tabular}


dose or long term systemic glucocorticoid therapy are the contributing factors [10]. Glucocorticoid induced osteoporosis is characterized by increased apoptosis of osteoblasts and osteocytes, thereby resulting in decreased bone formation and disruption of bone remodeling regulation. There is also increased osteoclast survival after initiation of glucocorticoid therapy, leading to increased bone resorption.

This initial increase in bone resorption is followed by a subsequent reduction of bone formation, leading to decreased peak bone mass and increased fracture risk [11]. A recent longitudinal study by the Canadian steroid-associated osteoporosis in the pediatric population (STOPP) consortium reported a $6 \%$ incidence of vertebral fractures 12 months after initiation of steroids [12]. The same group had previously documented a $7 \%$ prevalence of vertebral fractures within 30 days of initiating steroids. Most children in this cohort were asymptomatic, thereby stressing the need for early monitoring and appropriate investigations to detect fractures. Despite current knowledge, a survey to assess practices of North American pediatric rheumatologists regarding monitoring, prevention and treatment of BMD in children on long term steroid therapy showed that most respondent do not screen for low BMD on a regular basis, and only $35-50 \%$ of respondents prescribed bisphosphonates variably for osteoporosis in this population [13].

Management of secondary osteoporosis involves appropriate control of underlying inflammation, ensuring adequate calcium intake, attention to Vitamin D status, tapering and withdrawal of corticosteroids when possible, and timely physiotherapy to improve mobility. Prophylactic therapy with bisphosphonates is not indicated in children with low BMD. However, in clearly proven osteoporosis, there is evidence to suggest that bisphosphonates have a promising role.

Rationale and evidence for use: A 2006 systematic review of effectiveness and safety of bisphosphonates in treatment of low BMD and fractures JIA identified sixteen studies with 78 patients for effectiveness review [14]. Although the quality of studies was variable and there was no direct comparison of the intervention with control groups, in all studies, treatment with bisphosphonates uniformly increased BMD compared with baseline: the mean percentage increase in spine BMD ranged from $4.5 \%$ to
$19.1 \%$. There was also considerable variation in the dose and schedule of bisphosphonates. More recently, a 2010 Cochrane review [15] identified six randomised controlled trials, two controlled clinical trials, and one prospective cohort study of children with secondary osteoporosis receiving bisphosphonate treatment. Three of these studies were in children with secondary osteoporosis due to rheumatological conditions. The earliest controlled clinical trial identified in this review is by Lepore et al. [16], in which 7 patients with systemic or polyarticular JIA were treated with oral disodium clodronate and compared to a control group of 6 patients. They reported an $8 \%$ increase in BMD (measured by CT scan) in the treatment group and 7\% decrease in control group, at 12 months compared with baseline. Subsequently, Bianchi et al. [17] in a prospective multicentre cohort treated 38 patients in the treatment group with oral alendronate weekly for one year. They reported that the percent change of BMD significantly increased in treatment group from baseline to 12 months $(\mathrm{P}<0.002)$ however, the difference with the control group was not statistically significant. A mixed randomized controlled trial including 22 patients with rheumatological and other chronic inflammatory conditions used weekly oral alendronate in the treatment group and reported a statistically significant increase in the absolute BMD value in the treatment compared to placebo group [18]. The dose and regimen of bisphosphonates used for secondary osteoporosis in pediatric rheumatology is largely derived from studies in Osteogenesis imperfecta. In general, Zoledronic acid $0.1 \mathrm{mg} / \mathrm{kg} /$ year in 2 divided doses or Pamidronate $9 \mathrm{mg} / \mathrm{kg} /$ year in 4-6 divided doses is recommended. The recommended dose range for Intravenous Pamidronate is $0.5-1.5 \mathrm{mg} / \mathrm{kg} / \mathrm{day}$ for 3 days every 2-6 months and for Alendronate is 1-2 $\mathrm{mg} / \mathrm{kg} /$ week orally for a total duration of 12 months [5,6]. However, there are no established guidelines for the most appropriate dosing regimen and agent for secondary osteoporosis in Pediatrics.

\section{CRMO spectrum}

Chronic recurrent multifocal osteomyelitis (CRMO) is an auto-inflammatory disorder commonly seen in the age group less than 18 years. Other forms of unifocal non-infectious inflammatory osteomyelitis such as Majeed 
syndrome, or those associated with skin manifestations such as SAPHO syndrome (synovitis, acne, pustulosis, hyperostosis and osteitis) lie along the CRMO spectrum with different levels of severity. The histology of CRMO spectrum disorders reveals inflammatory changes with increased osteoclasts and bone resorption in early lesions. As bisphosphonates inhibit bone resorption and have demonstrated anti-inflammatory action, they have been used for pain management and preventing progress of lesions in CRMO. A single centre prospective study from Canada included 9 patients with CRMO who were treated with intravenous Pamidronate until resolution of MRI documented bone inflammation. Pamidronate resulted in resolution of pain and MRI documented inflammation in all patients [19]. The dose and regimen used in this study was adapted from the schedule used in treating Osteogenesis imperfecta. There are several individual case-reports and small case series of successful use of pamidronate for CRMO. An individual case report described marked symptomatic and radiological improvement in a 14-year-old girl with CRMO with oral Alendronate [20]. The pathology of CRMO is poorly understood, however, local and systemic increase in Tumor necrosis factor alpha has been demonstrated in active disease. Some of the effects of bisphosphonates are proposed to be due to their ability to supress pro-inflammatory cytokines such as TNF alpha, IL-6 and IL-1.

\section{Calcinosis in Juvenile Dermatomyositis (JDM)}

JDM is a rare chronic inflammatory disease of children characterized by inflammation of muscles, skin and other organs. Calcinosis is a form of dystrophic calcification that occurs as a frequent complication in JDM, affecting sites overlying pressure points such as elbows, knees, digits and buttocks. There is no consistently effective treatment for calcinosis and in JDM. Bisphosphonates have been tried for JDM calcinosis in view of their bone-resorptive action. In a short case-series of 6 patients with JDM calcinosis from Turkey, 4 out of 6 patients showed resolution of calcinosis with early initiation of bisphosphonates combined with immunosuppressive therapy [21]. Another retrospective review from a single-centre in India to determine the prevalence of calcinosis in children with JDM identified 9 patients with calcinosis of which $4 / 6$ children treated with alendronate showed partial reduction in calcinosis [22]. In a recent electronic survey by CARRA regarding practices related to calcinosis in JDM, bisphosphonates were the most frequently used alternative agents by $73 \%$ of respondents [23]. Doses used have been similar to that in secondary osteoporosis.

\section{Complex regional pain syndrome Type1 (CRPS1)}

CRPS 1 is a disorder characterized by pain, swelling, and autonomic skin changes, the precise mechanisms of which remain largely unknown. One of the implicated pain mechanism in CRPS 1 is the activation of two groups of acid-sensing nociceptors (TRPV1 and ASICs) due to acidosis secondary to microvascular hypoxia. It is possible that bisphosphonates act on this pathway by decreasing acidosis in the bone microenvironment thereby leading to an analgesic effect [2]. As previously mentioned, bisphosphonates also decrease the production of tumor necrosis factor alpha and other proinflammatory mediators, which can work in CRPS 1. In four randomized controlled trials in adults, bisphosphonate therapy significantly improved pain and functional impairments in the affected limb. The pooled results of a metaanalyses indicate that bisphosphonates are the most effective treatment options for CRPS-I in adults if started within 12 months of onset [24]. The only paediatric data, however comes from a case-report describing a good response of an 11-year-old girl to pamidronate, with no recurrence 2 years later. Their use for this pediatric indication thus remains unclear, pending further studies.

\section{Leg-Calve-Perthe's disease (LCPD)}

LCPD or avascular osteonecrosis of the femoral head is a developmental bone condition affecting children between 4 to 10 years of age. The rationale for using bisphosphonates in LCPD is their action on inhibiting osteoclastic bone resorption, thereby potentially preventing femoral head deformity. A systematic review of studies on bisphosphonates in LCPD concluded that there is lack of clinical evidence to say that bisphosphonates decrease femoral head deformity in LCPD, however, all studies noted subjective improvement of pain and gait in patients [25]. Only in one study, bisphosphonates were started before the stage of collapse of osteonecrosis and 
reported prevention of femoral head deformity in nine of seventeen patients. An Australian group has recently published a protocol for open-label Zoledronic acid treatment of patients with unilateral LCPD [26]. Results from this and further RCTs are needed before definitive recommendations can be made regarding use of bisphosphonates in avascular necrosis.

\section{Adverse effects and monitoring}

Recent consensus guidelines published by the Australasian pediatric endocrine group (APEG) [5] for use of bisphosphonates in children include recommendations for monitoring of side effects.

Infusion-related side effects: The most common adverse effect of pamidronate infusion is a selflimiting acute phase response characterized by flu-like symptoms (fever, bone pains, myalgia, nausea/vomiting) within 24-48 hours after the infusion, lasting up to several days after. Bisphosphonate-induced hypocalcaemia can occur due to inhibition of bone resorption and can be exacerbated by a pre-existing vitamin $\mathrm{D}$ deficiency. These infusions related complications can be prevented by ensuring a few simple steps.

- Prior to infusion: Ensure serum vitamin D level >50 nmol/L, ensure adequate calcium intake throughout.

- Lower dose for the first ever infusion can minimize the symptoms of acute phase effect.

- Paracetamol, anti-nausea medications, and fluids help to reduce the severity of acute phase effects.

Long-term side effects

Osteonecrosis of the jaw: There are no published pediatric reports on osteonecrosis of the jaw secondary to bisphosphonate use. However, it is recommended to complete all invasive dental work before the first dose of pamidronate, and a 6-12 monthly dental review while on pamidronate, owing to concerns from adult reports of osteonecrosis of the jaw.

Teratogenic effects: No published reports have demonstrated significant effects on the fetus when bisphosphonates were used prior to conception. However, there are concerns of teratogenicity from animal models, and hence it is recommended that pregnancy be avoided for 12 months after a dose of bisphosphonate.
Also, all pubertal and post pubertal girls should have a pregnancy test prior to bisphosphonate administration.

Table 2 provides the list of investigations to be undertaken before the first dose of bisphosphonates.

Gastrointestinal side effects to oral formulations: Oral bisphosphonates such as Alendronate often cause symptoms such as dyspepsia, esophagitis, esophageal reflux, and gastritis, sometimes leading to discontinuation. Factors that may contribute to GI side effects of Alendronate are: Ingesting it with less than $180 \mathrm{ml}$ of water, taking it in a supine position, lying down after ingesting the tablet, continuing to take it after the onset of symptoms suggestive of esophagitis, and having pre-existing esophageal disorders. On account of this, Merck, the manufacturer of Alendronate revised the Summary of Product Characteristics (SmPC) to include the need to take alendronate with at least $6 \mathrm{oz}$ of water, not chew or suck the tablet, and to remain upright for at least $30 \mathrm{~min}$ after taking the medicine [27].

Areas for further research: While bisphosphonates are a promising drug for use in children with secondary osteoporosis, there is no long-term safety data in the pediatric population. The pharmacokinetic properties and evidence of long-term renal elimination necessitate cautious use in pediatrics until better evidence is available from larger and long duration studies. Only a handful of case reports have demonstrated no adverse fetal effects of bisphosphonates, and more better-quality studies are needed to make recommendations for their use in the child-bearing female population. Concerns due to occurrence of osteonecrosis of the jaw in adult reports also mandate further longterm pediatric safety studies. There are no international recommendations on the choice of agent and duration of therapy in pediatrics. Bisphosphonates are usually discontinued when

\begin{tabular}{|} 
Table 2. Monitoring prior to first dose. \\
Monitoring prior to first dose \\
Full blood count \\
Urea and electrolytes \\
Liver function tests \\
\hline $25-O H$ Vitamin D \\
Parathyroid hormone \\
\hline Calcium, magnesium, phosphate \\
Dental review
\end{tabular}


the optimal outcome is achieved or if adverse effects occur. Randomized controlled trials are also needed to determine the true efficacy of bisphosphonates in rarer conditions such as CRMO, calcinosis of JDM, and CRPS-1 in children.

\section{References}

1. Russell RG. Bisphosphonates: The first 40 years. Bone. 49, 2-19 (2011).

2. Fleisch H. Bisphosphonates: Mechanisms of Action. Endocrine. Reviews. 19(1), 80-100 (1998).

3. Maksymowych WP, Jhangri GS, Leclercq $S$ et al. An open study of pamidronate in the treatment of refractory ankylosing spondylitis. J. Rheumatol. 25, 714-717 (1998).

4. Ghilardi JR, Ro" hrich H, Lindsay TH et al. Selective blockade of the capsaicin receptor TRPV1 attenuates bone cancer pain. J. Neurosci. 25, 3126-31 (2005).

5. Simm PJ, Biggin A, Zacharin $M$ et al. on behalf of the APEG Bone Mineral Working Group. Consensus guidelines on the use of bisphosphonate therapy in children and adolescents. J. Paediatr. Child. Health. 54 (2018) 223-233.

6. Baroncelli G, Bertelloni S. The Use of Bisphosphonates in Pediatrics. Horm. Res. Paediatr. 82, 290-302 (2014).

7. Papapoulos SE, Cremers SC. Prolonged bisphosphonate release after treatment in children. N. Engl. J. Med. 356, 1075-6 (2007).

8. Bishop N, Arundel P, Clark E et al. Fracture prediction and the definition of osteoporosis in children and adolescents: the ISCD 2013 Pediatric Official Positions. J. Clin. Densitom. 17(2), 275-80 (2014).

9. Henderson CJ, Cawkwell GD, Specker BL et al. Predictors of total body bone mineral density in non-corticosteroid treated prepubertal children with juvenile rheumatoid arthritis. Arthritis. Rheum. 40(11), 1967-1975 (1997).

10. McDonagh JE. Osteoporosis in juvenile idiopathic arthritis. Curr. Opin. Rheumatol. 13, 399-404 (2001).

11. Buehring B, Viswanathan R, Binkley $\mathrm{N}$ et al. Glucocorticoid-induced osteoporosis: An update on effects and management. J. Allergy. Clin. Immunol. 132(5), 1019-1030 (2013).

12. Rodd C, Lang B, Ramsay $\mathrm{T}$ et al. Incident vertebral fractures among children with rheumatic disorders 12 months after glucocorticoid initiation: a national observational study. Arthritis. Care. Res (Hoboken). 64(1), 122-31 (2012).

13. Soybilgic A, Tesher M, Wagner-Weiner L et al. A survey of steroid-related osteoporosis diagnosis, prevention and treatment practices of pediatric rheumatologists in North America. Pediatric. Rheumatology. 12(24), 1-6 (2014).
14. Thornton J, Ashcroft D, Mughal M et al. Systematic review of effectiveness of bisphosphonates in treatment of low bone mineral density and fragility fractures in juvenile idiopathic arthritis. Arch. Dis. Child. 91, 753761 (2006).

15. Ward L, Tricco A, Phuong PN et al. Bisphosphonate therapy for children and adolescents with secondary osteoporosis. Cochrane. Database. Syst. Rev. (4), CD005324 (2007)

16. Lepore L, Pennesi M, Barbi E et al. Treatment and prevention of osteoporosis in juvenile chronic arthritis with disodium clodronate. Clin. Exp. Rheumatol. 9(Suppl 6), 33-5 (1991).

17. Bianchi ML, Cimaz R, Bardare $M$ et al. Efficacy and safety of alendronate for the treatment of osteoporosis in diffuse connective tissue diseases in children: a prospective multicenter study. Arthritis \& Rheumatism. 43(9), 1960-6 (2000).

18. Rudge S, Hailwood S, Horne A et al. Effects of once-weekly oral alendronate on bone in children on glucocorticoid treatment. Rheumatology. 44(6), 813-8 (2005).

19. Miettunen P, Wei X, Kaura D et al. Dramatic pain relief and resolution of bone inflammation following pamidronate in 9 pediatric patients with persistent chronic recurrent multifocal osteomyelitis (CRMO), Pediatric. Rheumatology. 7, 2 (2009).

20. Hirano D, Chiba K, Yamada S et al. Oral alendronate in pediatric chronic recurrent multifocal osteomyelitis. Pediatrics. International. 59, 506-508 (2017).

21. Tayfur A, Topaloglu R, Gulhan B et al. Bisphosphonates in juvenile dermatomyositis with dystrophic calcinosis. Mod. Rheumatol. 25(4), 615-620 (2015).

22. Saini I, Kalaivani M, Kabra S. Calcinosis in juvenile dermatomyositis: frequency, risk factors and outcome. Rheumatol. Int. 36, 961-965 (2016).

23. Orandi A, Baszis K, Dharnidharka V et al. Assessment, classification and treatment of calcinosis as a complication of juvenile dermatomyositis: a survey of pediatric rheumatologists by the childhood arthritis and rheumatology research alliance (CARRA). Pediatr. Rheumatol. Online. J. 15, 71 (2017).

24. Chevreaua M, Romanda X, Gaudina P et al. Bisphosphonates for treatment of Complex Regional Pain Syndrome type 1: A systematic literature review and meta-analysis of randomized controlled trials versus placebo. Joint. Bone. Spine. 84, 393-399 (2017).

25. Young M, Little D, Kim H. Evidence for Using Bisphosphonate to Treat Legg-Calve'-Perthes Disease. Clin. Orthop. Relat. Res. 470, 2462-2475 (2017).

26. Jamil K, Zacharin M, Foster B. Protocol for a randomised control trial of bisphosphonate (zoledronic acid) treatment in childhood femoral head avascular necrosis due to Perthes disease. BMJ. Paediatr. Open. 1(1), e000084 (2017).

27. Merck Sharp \& Dohme. Summary of Product Characteristics (partial revision of text) fosamax (alendronate sodium MSD) (1996). 